\title{
ON THE MAXIMAL INEQUALITIES FOR CONDITIONAL DEMIMARTINGALES
}

\author{
Xinghui Wang and Shuhe Hu
}

Abstract. In this paper, based on a Fubini formula of conditional expectation and a maximal inequality for conditional demimartingales, we extend some inequalities of demimartingales to the case of conditional demimartingales. Meanwhile, we obtain some maximal $\phi$-inequalities for conditional demimartingales and some maximal inequalities of concave Young functions for conditional demimartingales.

Mathematics subject classification (2010): 60E15, $60 \mathrm{G} 48$.

Keywords and phrases: Conditional demimartingale, maximal $\phi$-inequalities, concave Young function, Doob's type inequality.

\section{REFERENCES}

[1] N. K. AgbeKo, Concave function inequalities for sub-(super-)martingales, Ann. Univ. Sci. Budapest, Sect. Math. 29 (1986), 9-17.

[2] G. Alsmeyer, U. Rösler, Maximal $\phi$-inequalities for nonnegative submartingales, Theory Probab. Appl. 50, 1 (2006), 118-128.

[3] Y. S. Chow, H. TeICHER, Probability Theory: Independence, Interchangeability, Martingales, 2nd ed. Springer-Verlag, New York, 1988.

[4] T. C. Christofides, Maximal inequalities for demimartingales and a strong law of large numbers, Statist. Probab. Lett. 50, 4 (2000), 357-363.

[5] T. C. Christofides, U-statistics on associated random variables, J. Statist. Plann. Inference 119, 1 (2004), 1-15.

[6] T. C. Christofides, M. HADJIKYRIAKOU, Maximal and moment inequalities for demimartingales and N-demimartingales, Statist. Probab. Lett. 82, 3 (2012), 683-691.

[7] T. C. Christofides, M. Hadjikyriakou, Conditional demimartingales and related results, J. Math. Anal. Appl. 398, 1 (2013), 380-391.

[8] J. Esary, F. Proschan, D. Walkup, Association of random variables with applications, Ann. Math. Statist. 38, 5 (1967), 1466-1474.

[9] X. B. Gong, Maximal $\phi$-inequalities for demimartingales, J. Inequal. Appl. 2011, 59 (2011).

[10] M. HADJIKYRIAKOU, Probability and moment inequalities for demimartingales and associated random variables, Ph. D. Dissertation, University of Cyprus, 2010.

[11] S. H. Hu, X. H. WAng, W. Z. YAng, X. J. WANG, Some inequalities for demimartingales and $N$-demimartingales, Statist. Probab. Lett. 82, 2 (2012), 232-239.

[12] C. M. Newman, A. L. WRIGHT, Associated random variables and martingale inequalities, $\mathrm{Z}$. Wahrsch. Verw. Geb. 59, 3 (1982), 361-371.

[13] B. L. S. PRAKASA RAO, On some maximal inequalities for demisubmartingales and N-demisupermartingales, J. Inequal. Pure Appl. Math. 8, 4 (2007), Article 112.

[14] B. L. S. PraKASA RAO, Conditional independence, conditional mixing and conditional association, Ann. Inst. Stat. Math. 61, 2 (2009), 441-460.

[15] B. L. S. PRAKASA RAO, Remarks on maximal inequalities for non-negative demisubmartingales, Statist. Probab. Lett. 82, 7 (2012), 1388-1390.

[16] B. L. S. Prakasa RAO, Associated Sequences, Demimartingales and Nonparametric Inference, Springer Basel AG, 2012.

[17] G. G. Rouss As, On conditional independence, mixing, and association, Stoch. Anal. Appl. 26, 6 (2008), 1274-1309. 
[18] J. F. WANG, Maximal inequalities for associated random variables and demimartingales, Statist. Probab. Lett. 66, 3 (2004), 347-354.

[19] X. H. WANG, X. J. WANG, Some inequalities for conditional demimartingales and conditional Ndemimartingales, Statist. Probab. Lett. 83, 3 (2013), 700-709.

[20] X. J. WAng, S. H. Hu, Maximal inequalities for demimartingales and their applications, Sci. China Ser. A, Math. 52, 10 (2009), 2207-2217.

[21] X. J. Wang, S. H. Hu, T. Zhao, W. Z. YAng, Doob's type inequalities and strong law of large numbers for demimartingales, J. Inequal. Appl. 2010, Art. ID 838301, 11 pp.

[22] X. J. WAng, B. L. S. Prakas a Rao, S. H. Hu, W. Z. YAng, On some maximal inequalities for demimartingales and $N$-demimartingales based on concave Young functions, J. Math. Anal. Appl. 396, 2 (2012) 434-440.

[23] D. M. YUAN, Y. K. YANG, Conditional versions of limit theorems for conditionally associated random variables, J. Math. Anal. Appl. 376, 1 (2011), 282-293. 